\title{
Video Analysis Techniques for Calculating the Coefficient of Friction of Ski Wax and Ski Base Treatments Using Miniature Ski Sleds
}

Jeffrey Bates ( $\sim$ jeff.bates@utah.edu )

University of Utah

Aaron Dobron

University of Utah

Kelan Albertson

University of Utah

Andrew Falkowski

University of Utah

Dennis Pruzan

University of Utah

Research Article

Keywords:

Posted Date: January 27th, 2022

DOI: https://doi.org/10.21203/rs.3.rs-1277300/v1

License: (9) This work is licensed under a Creative Commons Attribution 4.0 International License.

Read Full License 


\section{Abstract}

Testing the properties of ski base treatments is a challenging task because there are many environmental conditions and user preferences associated with both the testing and performance outcomes. In this work, we present a method for testing the coefficient of friction, and how it changes over repeated ski runs. We develop a ski track system to control the slope and geographic topography of the testing site, and we use video analysis techniques to calculate the change in the coefficient of friction across three ski runs. We have found an increase in the coefficient of friction after repeated ski runs, which indicates that there is some abrasion of the ski base treatments with repeated testing.

\section{Introduction}

Ski base treatments, including ski waxes and other alternative ski base treatments, are used by competitive, professional, and hobby skiers to enhance the glide performance of the ski base. From a physical perspective, glide performance is governed by friction at the ski-snow interface. Many ski waxes contain paraffin materials as their base, and even those that do not, still contain molecules of relatively low molecular weight. In both cases, the molecules are melted and applied to the ski base. Because of their high susceptibility to changes in thermal conditions, ski waxes and other ski base treatments have the potential for extreme changes in their material properties as the external temperature. Furthermore, ski conditions, including snow structure, snow surface temperature, air temperature, water content, and others, greatly impact the performance of skis and ski bases. [1-10] To mitigate some of the temperature-dependent properties of ski waxes, manufacturers have included additives into their wax compositions that enhance glide performance, and some have even specialized their waxes to specific temperature conditions, including low temperature waxes, high temperature waxes, and all-temperature waxes. $[1-4,11-13]$

The characterization of ski waxes and ski base treatments includes several limitations. First, the data obtained in a laboratory setting, even if it is controlled, does not include all of the environmental conditions that may be present during skiing.[14-31] Second, the environmental conditions can change suddenly during skiing, which will result in a change in ski base properties. Finally, the data obtained in the field is highly subjective when it is collected from individual skiers because it is based on the feel of skis, and different skiers have different material property preferences. [14, 15, 32-41]

Most published research includes the testing of glide performance, which can be defined as the coefficient of friction, all of which has determined that measuring and reporting the coefficient of friction is complex, and can include many different testing methods. Some studies have tested the coefficient of friction as skiers are towed across the snow surface, and others have tested the inertial measurement units to determine the coefficient of friction. In all studies, researchers have determined that the coefficient of friction is a function of both the ski base morphology and the surface roughness present in snow samples. $[16,17]$ 
To compare data obtained in a laboratory setting of the adhesion of ski wax to ski bases and the feel of the ski wax glide performance to data obtained in the field, this project explores the glide testing of ski base treatments under monitored environmental conditions with a controlled slope and track, where we built a snow track that remained as a permanent outdoor fixture and contained a controlled initial slope with a controlled run-out at the end of the track. In these experiments, we monitored the environmental conditions present during each experiment, and used video analysis techniques to calculate the coefficient of friction.

\section{Materials And Methods}

To test the coefficient of friction on various ski base treatments under monitored environmental conditions with a controlled slope and track, we selected a paraffin-free alternative material, in addition to the $\mathrm{CH} 7$ ski wax produced by manufacturer Swix. The ski base treatments were applied to stone ground ski bases according to manufacturer instructions on using a Swix Wax Iron. To obtain numerical data on the ski performance, we built a modular track system that could be set up in a variety of sites with a wide range of conditions (see Figure 1). The non-sloped portion of the track measured $30.8 \mathrm{~m}(100 \mathrm{ft}$ ) and had

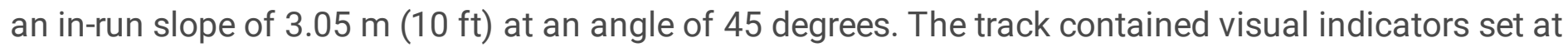
$7.62 \mathrm{~m}(25 \mathrm{ft})$ increments to help with video analysis by measuring the decrease in velocity over certain portions of the track. The snow surface of the track was groomed prior to testing, and was groomed between each ski set test. We tested seven runs of up to seven different ski sets that were treated with a variety of ski base treatments. For this study, we tested ski sets at four different test sites under a variety of environmental conditions. A constant weight was applied to each ski set, where the weight was distributed evenly and was attached to each ski set. Three different ski base treatments were tested in this study, which included three ski base treatments, including ski wax, a ski wax alternative material, and the ski wax in combination with the ski wax alternative material. Ski bases were prepared according to manufacturer instructions, where the skis were wiped with a base cleaner and allowed to dry for 5-10 minutes. The ski base treatment was applied, allowed to sit on the base, and once cooled or cured, the excess material was scraped from the ski base. The bases were further cleaned with a roto brush to clear the base treatment materials from the manufacturer-created surface topology on the ski bases.

A video camera was set up in a neutral position approximately $15.24 \mathrm{~m}(50 \mathrm{ft})$ away from the center point of the track runout to record the acceleration profile of each sled. The proximity to the test track produces

Figure 2. The side view shows ski sled traveling across the test track where time stamps were recorded as the sled marker traveled across each of the 5 stationary markers. The top view gives approximate distances between run-in/ run-out zones, video capture zone, stationary markers and video camera box.

the optimal field of view to reduce image distortion from the video camera. Each video was analyzed using a tracking software, which uses a reference point, specifically a florescent dot centered on the test sled, on a moving object relative to known distances at $7.62 \mathrm{~m}$ or $25 \mathrm{ft}$ increments on a track to yield a 
position vs. time profile that was subsequently analyzed to derive acceleration and coefficient of friction data. A visual representation of the video setup is shown in Figure 2.

\section{Results}

The initial data analysis yielded position vs. time curves for each run. An example position versus time curve generated from the analysis is shown in Figure 3. In order to extract coefficients of friction from this curve, the data set was broken into smaller subsets, shown by a unique color in Figure 3 . Each subset spans approximately $0.5 \mathrm{~m} / \mathrm{s}$ of deceleration. Each subset is subsequently fit to a 2 nd order polynomial, where the equations are shown next to the curve in Figure 3 in order to calculate the velocity and acceleration directly, and thereby the coefficients of friction for each run. Each individual run shown in Figure 3 yielded 3-4 data points, with 7-10 runs per treatment.

The resulting coefficients of friction were plotted in the $y$-axis and the average velocity in the $x$-axis, where the average velocity was calculated from each data subset, and is shown below in Figure 4, which includes a $95 \%$ confidence interval brackets. We initially plotted the three treatments against each other, but we were unable to determine a statistical difference between any of the three treatments. We further analyzed the data by plotting the measurable increase in friction for all treatments between the $1 \mathrm{st}$ run and after a $914 \mathrm{~m}(3000 \mathrm{ft})$ descent. The results show that the friction due to environmental changes over our testing period was much greater than the differences in friction between any of the three treatments and that our system does not currently have the resolution to differentiate between the treatments that we chose.

\section{Discussion}

These results show promise as a methodology, but there are some clear improvements that can be made in our approach. Firstly, we need to calculate real-time acceleration data from the sled itself instead of an independent sensor as the latter is susceptible to lens distortion and perspective distortion, which will induce small inaccuracies in the position vs. time data. We clearly see an increase in calculated coefficients of friction between the as-treated skis and those following a $914 \mathrm{~m} \mathrm{(3000} \mathrm{ft)} \mathrm{descent,} \mathrm{but} \mathrm{it} \mathrm{is}$ difficult to attribute that increase to the treatment itself; rather, it is likely the increase in ambient temperature, which increased by $5-10^{\circ} \mathrm{F}$ over the course of testing, and/or variability in snow type that resulted in the measurable increase in friction. However, the measured friction of all three treatments increased uniformly during the testing window and we can say that within our resolution, the treatments were indistinguishable from one another.

Regardless of the reported conditions, the process is repeatable and quickly yields relatively large data sets to calculate coefficient of friction vs. velocity curves. One must be conscious of the goal when choosing a test day. If the goal is to measure pure degradation in glide treatment, the experiment must be repeated on a day with little to no fluctuation in temperature throughout the day, thereby minimizing environmental effects on the results. However, if the goal is to measure the robustness of a specific 
treatment, a day which experiences a natural temperature fluctuation may yield more valuable information on how a specific glide treatment could perform over the course of the day moving through variable conditions.

From our preliminary experiments, we used single skis, and therefore obtained unreliable results. For example, there were inconsistencies among each of the individual mini skis, where each ski had a different length and width. In addition, there were no edges on the skis created for this experiment, which created uneven and varying ski edges and non-linear ski travel. Inconsistent ski base materials were used, where some had obvious imperfections or different grain structures in the raw UHMWPE. By using only one ski, the sleds typically tipped over or traveled in a non-linear pathway, and the single ski created an inability to distribute the weight evenly across the ski or to test multiple skis with the same methods and obtain the same results. The pathway was also modified due to non-linear sliding. Finally, there were many challenges with developing the video analysis methods and analyzing the ski travel in a non-linear direction. To overcome these challenges, we developed a weighted ski testing method, where the results are described above.

The weighted sled testing yielded more consistent results. There were still some challenges, including difficulty with uniform testing because of the difficulty in matching the weight distribution across sleds. When the weight distribution was uneven, the ski sled would travel in a non-linear pathway and leave the track. The environmental conditions were variable during each ski run because of the duration of setting each experiment up. We created eight pairs of weighted sleds with detachable weights that were uniformly applied to each ski sled pair. The video analysis method proved to be accurate in calculating the deceleration and resulting coefficient of friction. We also developed a reliable procedure for repeatable testing, including a test ramp, test track, appropriate spacing of pole measurements, level track, and weighted test sleds that could be attached to any ski sled specimen. Therefore, we created a sled testing procedure that allowed for the utilization of video analysis techniques to calculate the coefficient of friction between different ski sleds. In future experiments we will optimize our methods further with regard to the video analysis. A majority of the data was entered manually, leading to a margin of error. We also plan to create a stationary test track that remains consistent for each day testing occurs, rather than having to create a new test track and find new locations for each testing, which would decrease the error further.

\section{Conclusions}

The aim of this study was to create a controlled experimental setup to determine the coefficient of friction of ski base treatments to compare field data to data obtained in a laboratory setting. This project explored the glide testing of ski base treatments under monitored environmental conditions with a controlled slope and track, where we built a snow track that remained as a permanent outdoor fixture and contained a controlled initial slope with a controlled run-out at the end of the track. The resulting data demonstrates that video analysis techniques can be used to calculate the coefficient of friction between different ski sleds and that the coefficient of friction increases with progressive runs down the track. The 
results demonstrate that we can measure differences in coefficient of friction as small as 0.01 , which is sensitive enough to measure large environmental changes but not small differences in glide between base treatments, which can be as small as 0.001 .

\section{Declarations}

\section{Acknowledgements}

The researchers would like to thank the Materials Characterization Lab at the University of Utah for the use of their characterization equipment.

\section{References}

1. Rogowski, l., et al., Typology of the gliding waxes in cross-country skiing: Comparison between classifications based on the chemical composition and those based on the physical and physicochemical properties. Cold Regions Science and Technology, 2005. 43(3): p. 140-149.

2. Rogowski, l., et al., Influence of fluorine-based additive content on the physical and physicochemical properties of ski gliding wax. Cold Regions Science and Technology, 2007. 49(2): p. 145-150.

3. Rohm, S., et al., Wear of ski waxes: Effect of temperature, molecule chain length and position on the ski base. Wear, 2017. 384: p. 43-49.

4. Kuzmin, L. and M. Tinnsten, Hot Glide Wax Treatment and the Hardness of the Ski Running Surface (P163), in The Engineering of Sport 7. 2008. p. 135-141.

5. Bowden, F.P., Friction on snow and ice. Proceedings of the Royal Society of London. Series A. Mathematical and Physical Sciences, 1953. 217(1131): p. 462-478.

6. Bäurle, L., Sliding friction of polyethylene on snow and ice. 2006, ETH Zurich.

7. Kuzmin, L., Interfacial kinetic ski friction. 2010, Mittuniversitetet.

8. Kuroiwa, D., The kinetic friction on snow and ice. Journal of Glaciology, 1977. 19(81): p. 141-152.

9. Rohm, S., et al., Effect of Different Bearing Ratios on the Friction between Ultrahigh Molecular Weight Polyethylene Ski Bases and Snow. ACS Appl Mater Interfaces, 2016. 8(19): p. 12552-7.

10. Theile, T., et al., Mechanics of the ski-snow contact. Tribology letters, 2009. 36(3): p. 223-231.

11. Abishek, S., et al., Effect of microstructure on melting in metal-foam/paraffin composite phase change materials. International Journal of Heat and Mass Transfer, 2018. 127: p. 135-144.

12. Mondal, S., Phase change materials for smart textiles-An overview. Applied thermal engineering, 2008. 28(11-12): p. 1536-1550.

13. Jamekhorshid, A., S. Sadrameli, and M. Farid, A review of microencapsulation methods of phase change materials (PCMs) as a thermal energy storage (TES) medium. Renewable and Sustainable Energy Reviews, 2014. 31: p. 531-542. 
14. Cousins, I.T., et al., The concept of essential use for determining when uses of PFASs can be phased out. Environmental Science: Processes \& Impacts, 2019. 21(11): p. 1803-1815.

15. Vierke, L., et al., Perfluorooctanoic acid (PFOA)-main concerns and regulatory developments in Europe from an environmental point of view. Environmental Sciences Europe, 2012. 24(1): p. 16.

16. Swarén, M., et al., Validation of test setup to evaluate glide performance in skis. Sports Technology, 2015. 7(1-2): p. 89-97.

17. Buhl, D., M. Fauve, and H. Rhyner, The kinetic friction of polyethylen on snow: the influence of the snow temperature and the load. Cold Regions Science and Technology, 2001. 33(2-3): p. 133-140.

18. Breitschädel, F., $\varnothing$. Lund, and S. Løset, Cross country ski base tuning with structure imprint tools. Procedia Engineering, 2010. 2(2): p. 2907-2911.

19. Kuzmin, L., Investigation of the most essential factors influencing ski glide. 2006, Luleå tekniska universitet.

20. Breitschädel, F., N. Haaland, and N. Espallargas, A Tribological Study of UHMWPE Ski Base Treated with Nano Ski Wax and its Effects and Benefits on Performance. Procedia Engineering, 2014. 72 : p. 267-272.

21. Breitschädel, F., A New Approach for the Grinding of Nordic Skis. Procedia Engineering, 2015. 112: p. 385-390.

22. Stamboulides, C., P. Englezos, and S.G. Hatzikiriakos, The ice friction of polymeric substrates. Tribology International, 2012. 55: p. 59-67.

23. Ekström, H. Future developments in cross-country skiing equipment. in Skiing Trauma And Safety: Fifth International Symposium. 1985. ASTM International.

24. Ducret, S., et al., Friction and abrasive wear of UHWMPE sliding on ice. Wear, 2005. 258(1-4): p. 2631.

25. Nachbauer, W., P. Schröcksnadel, and B. Lackinger, Effects of snow and air conditions on ski friction, in Skiing Trauma and Safety: Tenth Volume. 1996, ASTM International.

26. Krupa, I., G. Miková, and A. Luyt, Phase change materials based on low-density polyethylene/paraffin wax blends. European Polymer Journal, 2007. 43(11): p. 4695-4705.

27. Fischer, J., G.M. Wallner, and A. Pieber, Morphology of polyethylene ski base materials. J Sports Sci, 2010. 28(5): p. 555-62.

28. Nordin, N.A. and P. Styring, Superhydrophobic Ski Bases for Reduced Water Adhesion. Procedia Engineering, 2014. 72: p. 605-610.

29. Floros, M.C., L. Raghunanan, and S.S. Narine, A toolbox for the characterization of biobased waxes. European Journal of Lipid Science and Technology, 2017. 119(6).

30. Venkatesan, R., et al., The strength of paraffin gels formed under static and flow conditions. Chemical Engineering Science, 2005. 60(13): p. 3587-3598.

31. Nefedov, N., et al., Peculiarities of temperature behavior of low-molecular fluorooligomers. Polymer Science, Series A, 2017. 59(4): p. 496-505. 
32. Lee, K.-H. and S.C. Oh, Kinetics of the thermal degradation of wax materials obtained from pyrolysis of mixed waste plastics. Korean journal of chemical engineering, 2010. 27(1): p. 139-143.

33. Xu, B. and Z. Li, Paraffin/diatomite composite phase change material incorporated cement-based composite for thermal energy storage. Applied energy, 2013. 105: p. 229-237.

34. Chen, Z., et al., Synthesis and characterization of microencapsulated paraffin microcapsules as shape-stabilized thermal energy storage materials. Nanoscale and microscale thermophysical engineering, 2013. 17(2): p. 112-123.

35. Grzeskowiak, K., et al., Influence of waxes remelting used in investment casting on their thermal properties and linear shrinkage. Metalurgija, 2015. 54(2): p. 350-352.

36. Wikner, A., Tribological Behaviour of Newly Developed UHMWPE-nanocomposites: As Suitable Candidates for Ski Material and Design. 2015.

37. Breitschädel, F., Technical aspects to improve performance in cross-country skiing. 2014, PhD.

38. Gambaretto, G., et al., Synthesis and characterization of a new class of polyfluorinated alkanes: tetrakis (perfluoroalkyl) alkane. Journal of fluorine chemistry, 2003. 121(1): p. 57-63.

39. Wannasri, S., et al., Increasing wear resistance of UHMWPE by mechanical activation and chemical modification combined with addition of nanofibers. Procedia Engineering, 2009. 1(1): p. 67-70.

40. Chand, N., A. Naik, and H. Khaira, Development of UHMWPE modified PP/PET blends and their mechanical and abrasive wear behavior. Polymer composites, 2007. 28(2): p. 267-272.

41. Chang, T., C. Yuan, and Z. Guo, Tribological behavior of aged UHMWPE under water-lubricated condition. Tribology International, 2019. 133: p. 1-11.

\section{Figures}




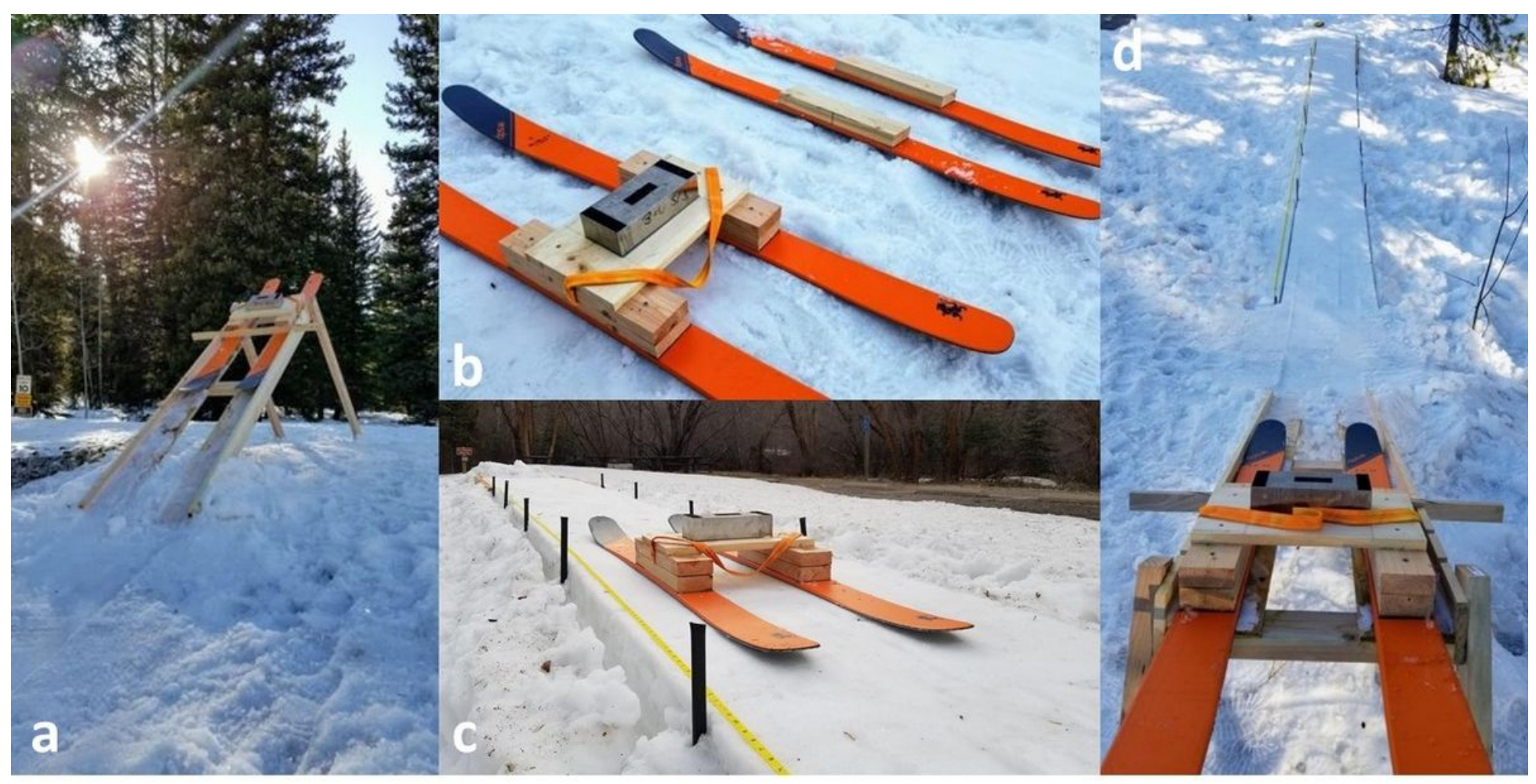

\section{Figure 1}

The ski track included an angled portion with a fixed angle that would allow for acceleration (a and d), the ski sleds were built to allow for fixed and uniform weight distribution (b), and the ski track included a flat section with no gradient to allow for deceleration (c and d). 
SIDE VIEW
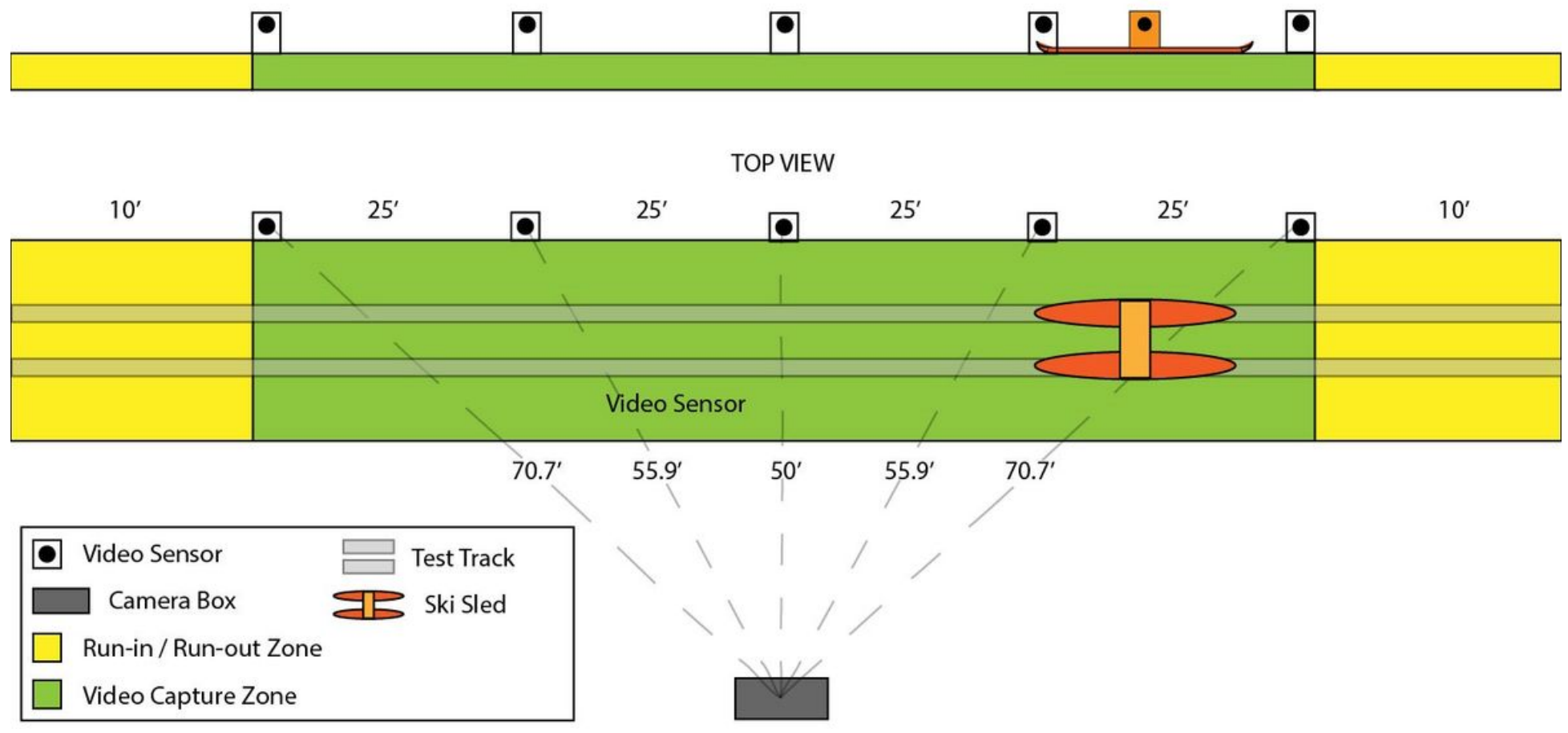

Figure 2

The side view shows ski sled traveling across the test track where time stamps were recorded as the sled marker traveled across each of the 5 stationary markers. The top view gives approximate distances between run-in/ run-out zones, video capture zone, stationary markers and video camera box. 


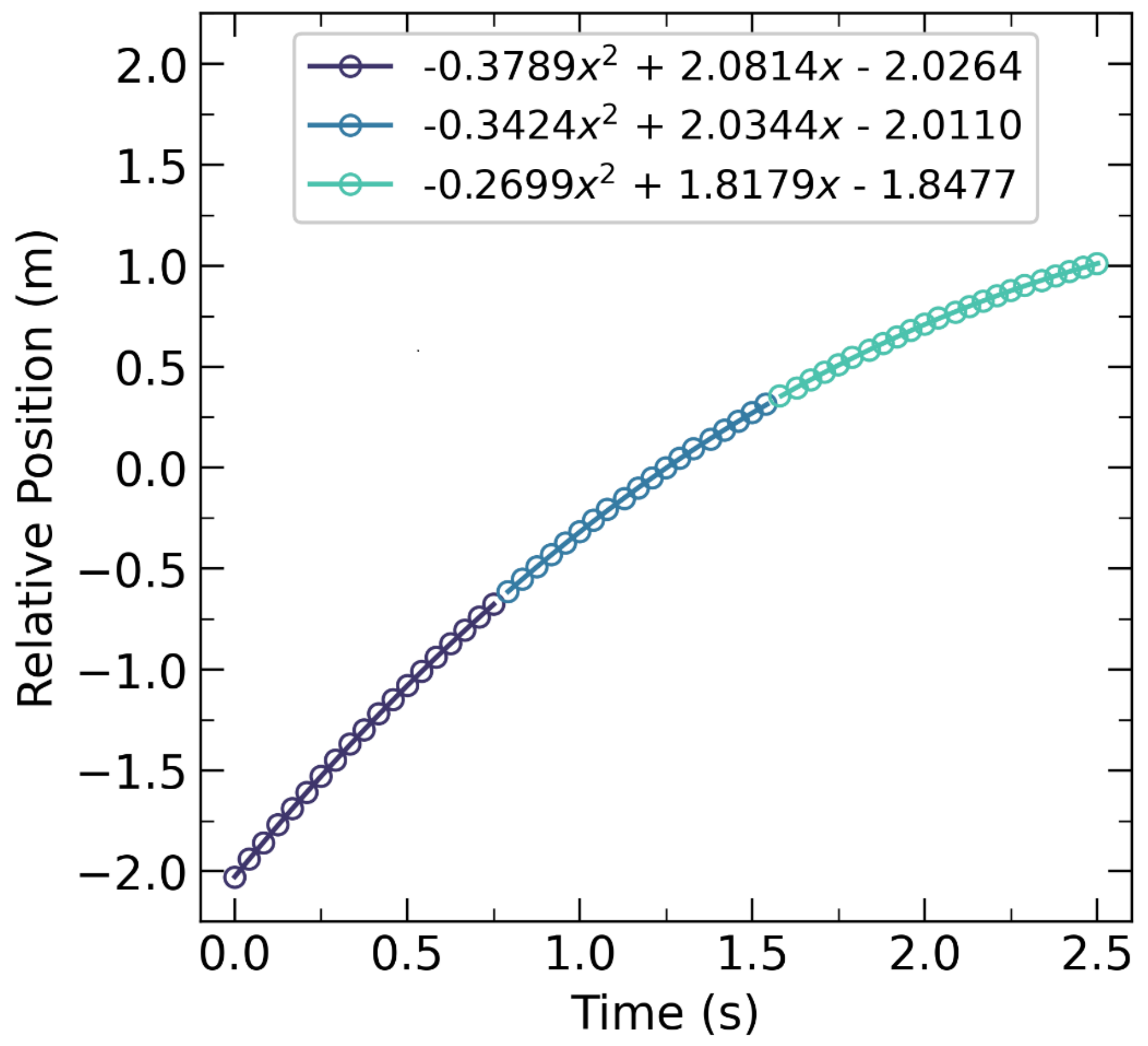

Figure 3

Position vs. Time curve for a test sled using "Treatment 1". The data set was broken into smaller subsets, each of which was fit to a 2 nd order polynomial, from which the acceleration and coefficient of friction were calculated. 


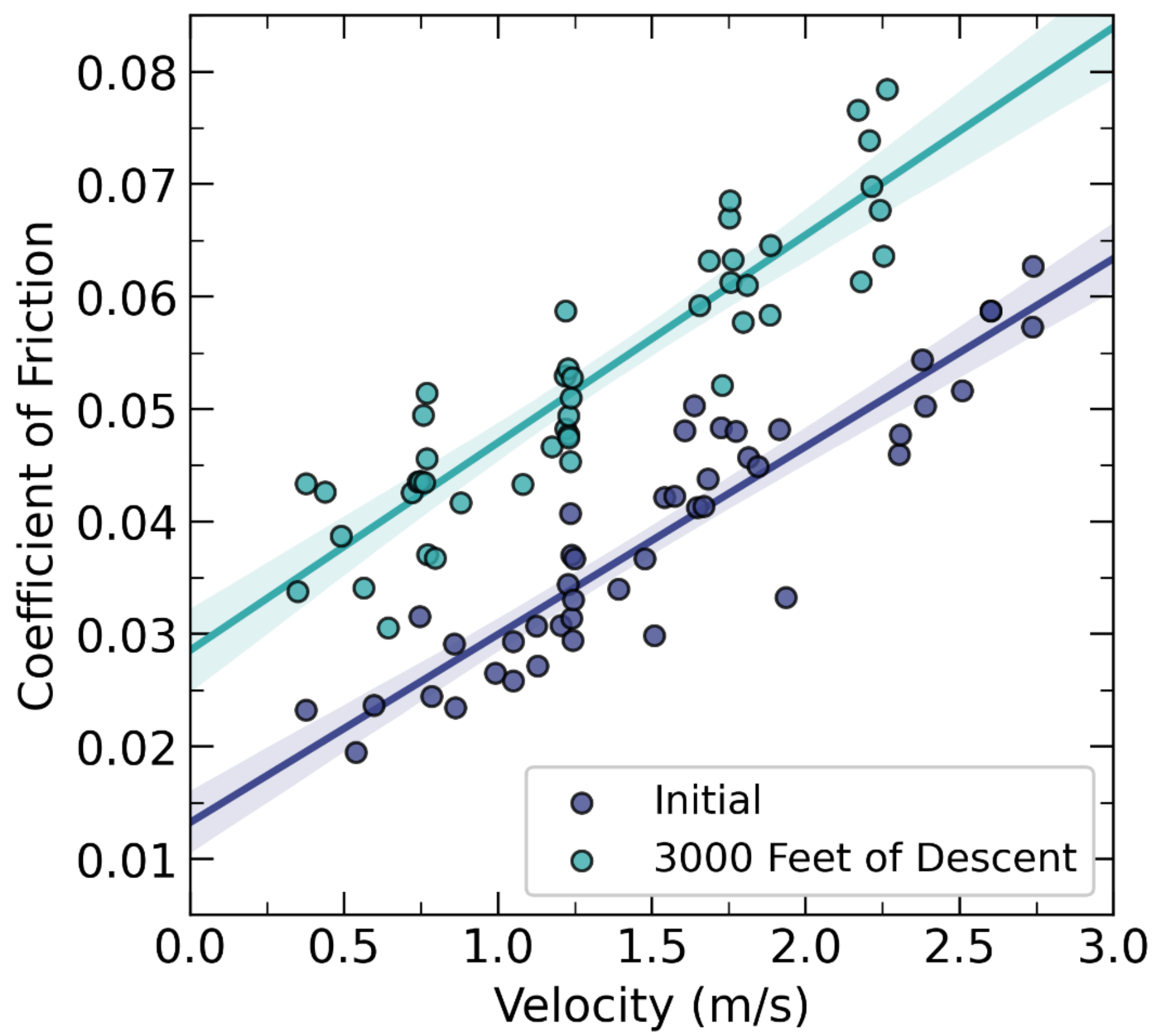

Figure 4

The change in the coefficient of friction of the ski sled was measured after 3000 feet of descent. The $95 \%$ confidence interval for each of the data sets is indicated by the highlighted regions. 